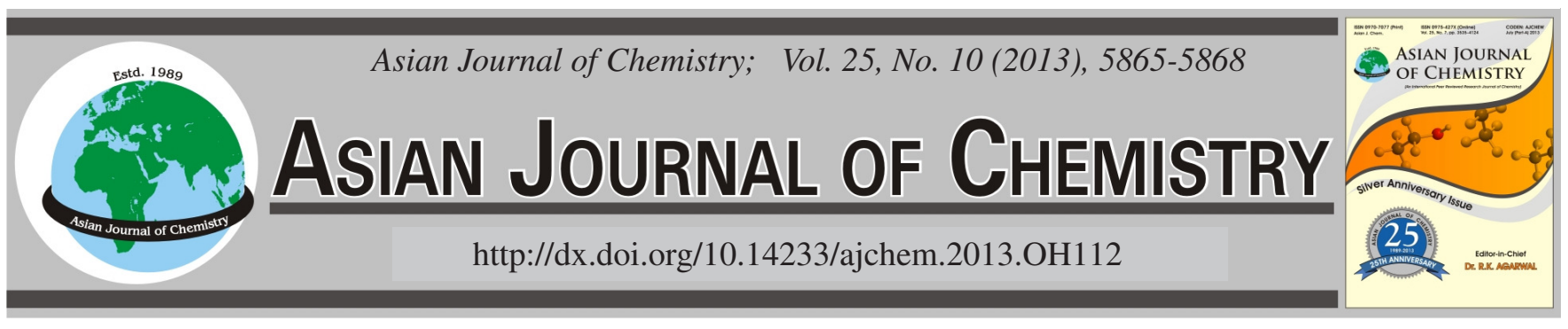

\title{
Emission Prediction of Municipal Solid Waste by Physical and Chemical Characteristics Analysis $\dagger$
}

\author{
Jin-Do ChUnG ${ }^{1}$, Jin-WAN KIM ${ }^{2}$ and SEUNG-Min HwANG ${ }^{3}, *$
}

\begin{abstract}
${ }^{1}$ Department of Environmental Engineering, Hoseo University, Asan city, Chung-nam 336-795, Republic of Korea
${ }^{2}$ Graduate School, Department of Environmental Engineering, Hoseo University, Asan city, Chung-nam 336-795, Republic of Korea

${ }^{3}$ The Faculty of Co-op, Hoseo University, Asan city, Chung-nam 336-795, Republic of Korea
\end{abstract}

*Corresponding author: Fax: +82 41 5405483; E-mail: hwangsm@hoseo.edu

\begin{abstract}
In this study, we investigated medium long-term physico-chemical characteristics of domestic waste in small and medium cities of Chungcheongnam-do, C city, A city and D gun. Apparent density, physical composition, three ingredients, elementary composition and caloric value of domestic waste of each area in winter season were analyzed in accordance with the waste process test method. The basic unit emission of domestic waste was calculated and future production was predicted. The result of analysis indicates that no difference in apparent density was shown by district and, as to physical composition, food in C city and A city and paper in D gun showed the highest percentages. The result of analyzing elementary composition showed that carbon content and oxygen content in D gun were lower than those of $\mathrm{C}$ city and A city. Also, in the analysis of caloric values, D gun was the lowest showing a value of $2188 \mathrm{kcal} / \mathrm{kg}$ in comparison to those of $\mathrm{C}$ city and A city which were $2,634 \mathrm{kcal} / \mathrm{kg}$ and $2,231 \mathrm{kcal} / \mathrm{kg}$, respectively. As to the basic unit emission, while there was no big difference between $\mathrm{C}$ city and A city which were $1.11 \mathrm{~kg} /$ day·person and $1.02 \mathrm{~kg} / \mathrm{day} \cdot$ person respectively, D gun was $0.63 \mathrm{~kg} / \mathrm{day} \cdot \mathrm{person}$ showing a big difference.
\end{abstract}

Key Words: Municipal solid waste, Emission prediction, Landfill, Physical-chemical characteristic.

\section{INTRODUCTION}

To process the wastes reasonably and to establish the wastes management policy, the most essential and basic stage is to determine the wastes emission and to analyze their properties. As the wastes emission is being increased by the development of industries and improvement of economic level, it requires new landfill sites or re-using existing landfill sites and expansion of wastes treatment facilities like incineration facilities, etc. Since the wastes are very uneven in quality and the physical and chemical characteristics are varied depending on the generated regions, it is hard to refer and apply directly the property data of the other regions ${ }^{1,2}$. Therefore, to establish the wastes management policy to process the wastes, the investigation on the physical and chemical characteristics of the domestic wastes by region should be preceded. The wastes management in the past was the concept of cleaning, which removes the rubbish filths for the health and hygiene of the urban residents and the processing method was depended on the simple landfill, the easiest and cheapest method. However, as the social structure is changed into the structures of urbanization, mass-production and mass-consumption, the problems accompanied by wastes management are being changed very complicatedly and diversely and to treat them with environmentally safe method, diverse methods are being attempted. Since the final objective of these methods is to minimize the amount of wastes emission at first hand, the comprehensive measures should be established such as processing wastes safely with low cost through development of wastes processing technologies, etc. . $^{3,4}$

In this study, the wastes emission, physical and chemical characteristics, caloric value, etc., are served as basic data to process the wastes with safe method, were identified from the small and medium sized C city, A city and D gun having 500 thousand, 200 thousand and 100 thousand populations respectively, in which the commercial, industrial and agricultural activities are made actively and compared each other.

\section{EXPERIMENTAL}

The experimental materials were collected from general domestic wastes thrown out in the standard plastic garbage bags and brought into landfill sites and incineration sites from the area of apartment houses, detached houses, industrial complex, public facilities and market area of $\mathrm{C}$ city, A city 
and D gun from December, 2009 to February, 2010, which were winter season and to avoid the wastes brought in to be concentrated in one area, the wastes samples were collected directly from each area before being collected by the local governments. The analysis items were fixed as physical composition, apparent density, three ingredients, elementary composition (analysis), caloric value and heavy metal ingredients. The standard plastic garbage bags were opened, the wastes were thrown on the vinyl sheet for tent and mixed several times in order to make garbage of $4 \mathrm{~m}^{3}$ and to be distributed evenly so that they could represent the wastes of that region. In addition, the sample having big volume were crushed in appropriate size and mixed together. The sample for laboratory analysis was collected from the garbage mixed several times during $20 \mathrm{~min}$ according to 4 division method by cone style. The samples were classified into 9 items; foods, papers, plastics, woods, rubbers-leathers, textiles, metals, glasses-ceramics, earthy materials and others. The weight ratio (wt \%) by composition obtained from the samples classified as such was calculated and used as physical composition. For the three ingredients, the samples were analyzed in order to show representability. For the chemical characteristics of the wastes, the composition of the ingredients for unknown sample was obtained by conducting qualitative and quantitative analyses by $\mathrm{C}, \mathrm{H}, \mathrm{N}, \mathrm{S}$ and $\mathrm{O}$, which are the major elements of organic compounds and the elementary analysis for the $\mathrm{C}$, $\mathrm{H}, \mathrm{O}, \mathrm{N}$ and $\mathrm{S}$ of the combustible materials were performed using automatic elemental analyzer, which is a device to infer the empirical formula. The samples burned at approximately $1,800{ }^{\circ} \mathrm{C}$ were resolved in the reaction vessel and separated according to the moving velocity as it passes the GS column and detected in TCD. To analyze the caloric value of the domestic wastes, the caloric value of the domestic wastes was calculated using bomb calorimeter (Parr, model No. 1261). In addition, the concentration of heavy metal within the domestic wastes was calculated using inductively coupled plasma mass spectrometer. The physical and chemical characteristics of the domestic wastes generated during winter time in A city, $\mathrm{C}$ city and $\mathrm{D}$ gun was compared and analyzed using the data obtained above.

\section{RESULTS AND DISCUSSION}

The analysis results by location of each region obtained from general domestic wastes collected were averaged and compared by region. The analysis results of the wastes property from C city, A city and D gun, which has 500 thousand, 200 thousand and 100 thousand populations respectively, are as follows.

Apparent density and physical composition: In this study, to find out physical and chemical characteristics of the domestic wastes by regions in Chungcheongnam-do during winter time, the physical composition, apparent density, three ingredients, elementary analysis, caloric value and heavy metal ingredients were examined. Table-1 showed that the mean apparent densities and physical compositions by the $\mathrm{C}$ city, $\mathrm{A}$ city and D gun. The apparent densities of A city, C city and D gun were measured as $0.19,0.20$ and $0.19 \mathrm{ton} / \mathrm{m}^{3}$ and did not show any distinctive regional characteristics.

\begin{tabular}{|c|c|c|c|c|c|}
\hline \multicolumn{6}{|c|}{$\begin{array}{c}\text { TABLE-1 } \\
\text { COMPARISON OF APPARENT DENSITY AND PHYSICAL } \\
\text { COMPONENTS OF MUNICIPAL SOLID WASTES IN } \\
\text { C CITY, A CITY AND D GUN }\end{array}$} \\
\hline \multicolumn{3}{|c|}{ Apparent density (ton $/ \mathrm{m}^{3}$ ) } & $\begin{array}{c}\text { C city } \\
\text { Average }\end{array}$ & $\begin{array}{c}\text { A city } \\
\text { Average }\end{array}$ & $\begin{array}{c}\text { D gun } \\
\text { Average }\end{array}$ \\
\hline \multirow{13}{*}{$\begin{array}{l}\text { Physical } \\
\text { composition } \\
\text { (\%) }\end{array}$} & \multirow{7}{*}{ Combustible } & Food & 26.9 & 22.3 & 15.4 \\
\hline & & Paper & 25.7 & 19.6 & 32.3 \\
\hline & & Plastic & 15.3 & 21.9 & 24.1 \\
\hline & & Wood & 9.0 & 3.4 & 3.1 \\
\hline & & $\begin{array}{l}\text { Rubber- } \\
\text { leather }\end{array}$ & 6.5 & 13.8 & 3.6 \\
\hline & & Fiber & 7.4 & 6.5 & 8.5 \\
\hline & & Sum & 90.8 & 87.5 & 87.0 \\
\hline & \multirow{6}{*}{$\begin{array}{c}\text { Non- } \\
\text { combustible }\end{array}$} & Metal & 2.8 & 5.0 & 3.5 \\
\hline & & Glass & 3.5 & 4.7 & 6.5 \\
\hline & & Sand & 0.7 & 0.9 & 0.4 \\
\hline & & Others & 2.2 & 1.9 & 2.5 \\
\hline & & Sum & 9.2 & 12.5 & 13.0 \\
\hline & & Total & 100 & 100 & 100 \\
\hline
\end{tabular}

In the physical composition of the wastes collected from A city, the foods were occupied the highest proportion of $22.34 \%$ followed by plastics $(21.94 \%)$, papers $(19.55 \%)$, rubbers.leathers $(13.81 \%)$, textiles $(6.45 \%)$ and woods $(3.42$ $\%$ ) in order and the combustibles were occupied $87.51 \%$ and nonflammables were $12.49 \%$. In the physical composition of the wastes collected from C city, the foods were occupied 26.91 $\%$ followed by papers $(25.74 \%)$, plastics (15.31\%), woods $(8.98 \%)$, textiles $(7.42 \%)$ and rubbers leathers $(6.48 \%)$, in order and the combustibles were occupied $90.84 \%$ and nonflammables were $9.16 \%$. In the physical composition of the wastes collected from D gun, the papers were occupied the highest proportion of $32.33 \%$ followed by plastics $(24.13$ $\%)$, foods $(15.37 \%)$, textiles $(8.47 \%)$, rubbers/leathers $(3.63$ $\%)$ and woods $(3.11 \%)$ in order and the combustibles were occupied $87.04 \%$ and nonflammables were $12.96 \%$. In all three regions, the foods, papers and plastic wastes account for major part and it was analyzed that they were occupied more than $60 \%$. It was observed that no distinctive difference was found in the physical composition. In case of $\mathrm{C}$ city and $\mathrm{A}$ city, which the economic and industrial activities are performed actively in large scale, the foods were occupied higher proportion and in case of D gun, which is in transition period, the foods were occupied the lowest proportion. It deemed that the reason that foods were occupied lowest in D gun was that they treated the food wastes by themselves because the agriculture is activated and in case of $\mathrm{C}$ city and A city, it deemed that the food wastes were occupied the higher proportion because no big sanction is imposed when they thrown out the food wastes in the general standardized garbage bags. In addition, it deemed that the proportion of the plastics wastes was low because the consumption of plastics such as PET, etc. was reduced during winter time.

Analysis of three ingredients: Three ingredients measured by each region are shown in Table-2. In case of $\mathrm{C}$ city, the water, combustibles and ash contents were represented as $34.69,56.6$ and $8.69 \%$ respectively and in the A city, the water, combustibles and ash contents were represented as $28.12,58.55$ and $13.33 \%$ respectively. As the water contents 


\begin{tabular}{clccc}
\hline \multicolumn{5}{c}{ TABLE-2 } \\
\multicolumn{4}{c}{ COMPARISON OF THREE COMPONENT ANALYSIS } \\
& OF MSW IN C CITY, A CITY AND D GUN \\
\cline { 3 - 5 } & & C city & A city & D gun \\
\cline { 3 - 5 } & & Average & Average & Average \\
\hline \multirow{2}{*}{ Three } & Moisture & 34.7 & 28.1 & 31.4 \\
component & Combustible & 56.6 & 58.6 & 57.6 \\
(wt \%) & Ash & 8.7 & 13.3 & 11.0 \\
& Sum & 100.0 & 100.0 & 100.0 \\
\hline
\end{tabular}

were represented low in general, it deemed to be caused by the influence of the season. Generally, the water contents represents higher in summer than winter. In D gun, the water, combustibles and ash contents were represented as 31.41 , 57.58 and $11.01 \%$ respectively. Among three regions, as the water content of domestic wastes from $\mathrm{C}$ city were highest and the ash contents were lowest, it deemed that they contained high proportion of food wastes and the contents of papers, woods, etc. were relatively high. In A city, that the water contents were lowest and the ash contents were highest is deemed that the contents of the foods and papers, which contain relatively high amount of water, were low while the rubber and leather contents were high. In case of D gun, it deemed that the overall contents of water represented high because the papers and plastics were contained the lots of water of the foods although the contents of foods were low. The reason that the food composition was low comparing with other regions is deemed to be related to economic level of the region because the scale of unit organized the region is small than other regions except the apartment houses. In the meantime, it showed the tendency that the contents of combustibles were lowered as the water content was growing high. If the separate collection of the food wastes, etc. is performed well, it deemed that the water contents of the domestic wastes are lowered and the quality of the garbage will be improved.

\begin{tabular}{|c|c|c|c|c|}
\hline \multicolumn{5}{|c|}{$\begin{array}{c}\text { TABLE-3 } \\
\text { COMPARISON OF CHEMICAL CHARACTERISTICS } \\
\text { OF MSW IN C CITY, A CITY AND D GUN }\end{array}$} \\
\hline & & $\begin{array}{c}\text { C city } \\
\text { Average }\end{array}$ & $\begin{array}{c}\text { A city } \\
\text { Average }\end{array}$ & $\begin{array}{c}\text { D gun } \\
\text { Average }\end{array}$ \\
\hline \multirow{7}{*}{$\begin{array}{l}\text { Elemental } \\
\text { analysis } \\
\text { (wt \%) }\end{array}$} & $\mathrm{C}$ & 45.8 & 45.2 & 39.3 \\
\hline & $\mathrm{H}$ & 6.9 & 6.1 & 5.7 \\
\hline & $\mathrm{O}$ & 30.3 & 29.4 & 24.6 \\
\hline & $\mathrm{N}$ & 0.8 & 1.1 & 0.6 \\
\hline & S & 0.1 & 0.1 & 0.2 \\
\hline & $\mathrm{Cl}$ & 0.3 & 0.5 & 0.3 \\
\hline & Ash & 16.2 & 17.7 & 29.3 \\
\hline
\end{tabular}

Elementary analysis: Elementary composition is important analysis related to the amount of combustion air when incinerating, control of atmospheric pollutants, etc. Generally, the higher the contents of carbon and hydrogen are, the property of the wastes is considered to be high quality. The elementary analysis results showed that in the elementary composition of each city, the carbon, oxygen and hydrogen are occupied the high proportion in order, which is the trend consistent with general elementary analysis of the wastes. In the $\mathrm{C}$ city and $\mathrm{A}$ city, the carbon was represented $45 \%$ and oxygen was represented as $30.03 \%$ and $29.36 \%$ respectively and the hydrogen was represented as $6 \%$ showing similar composition. In the elementary analysis for the D gun, the carbon, oxygen and hydrogen was represented 39.33, 24.64 and $5.67 \%$ respectively. If the elementary analysis is examined considering empirical formula to obtain caloric value, it can be predicted that the caloric value for D gun may be lower than that of $\mathrm{C}$ city and A city. This is because that the influence of the carbon contents on the caloric value occupied great portion. Generally, it is expected that the level of life and the quality of domestic wastes may be lower than those of $\mathrm{C}$ city and A city.

Analysis of caloric value: To analyze the caloric value of the wastes from each city, the caloric value was calculated using bomb calorimeter and the results are shown in Table-4. The caloric value analysis is the important to determine whether the design and installation of heat recovery facilities through the incineration of the wastes are required or not and is the data to determine the possibility of incineration (being used as fuel). However, since the actual incinerator, the water contained in the exhaust gas is discharge as vapour and the condensation heat is mostly not used, the low caloric value except the condensation heat of the water in the higher heating value is the important factor. In the caloric value analysis results, the low caloric value of the $\mathrm{C}$ city represent the highest as $2,634 \mathrm{kcal} / \mathrm{kg}$ and the A city and D gun were represented as $2,231 \mathrm{kcal} / \mathrm{kg}$ and $2,188 \mathrm{kcal} / \mathrm{kg}$ respectively. This was expected from result of above three ingredients analysis and elementary analysis. In case of D gun, since the papers and plastics were occupied the great proportion in the physical composition, it seems to have high caloric value but in the elementary composition of the wastes (for D gun), it is observed that the carbon contents are low. This seems that the wastes are contained not the sound papers and plastics but lots of foreign materials, for which it deemed that the caloric value was calculated low.

\begin{tabular}{|c|c|c|c|}
\hline \multicolumn{4}{|c|}{$\begin{array}{c}\text { TABLE-4 } \\
\text { HEATING VALUE OF MUNICIPAL SOLID WASTES } \\
\text { IN C CITY, A CITY AND D GUN }\end{array}$} \\
\hline & C city & A city & D gun \\
\hline & Average & Average & Average \\
\hline High heating value $(\mathrm{kcal} / \mathrm{kg})$ & 3,202 & 2,722 & 2,674 \\
\hline Low heating value $(\mathrm{kcal} / \mathrm{kg})$ & 2,631 & 2,233 & 2,186 \\
\hline
\end{tabular}

Waste emission and prediction: In the wastes emission, the characteristic by the population was observed. The $\mathrm{C}$ city has highest population and it is observed that the wastes emission per basic unit is also high. Although not so much differences are shown from A city, it has $70 \%$ higher than D gun, which has lowest population. In the aspects of the regional characteristics, the $\mathrm{C}$ city, in which the commercial and industrial activities are active, has highest emission per basic unit and the A city, in which the industrial and agricultural activities are active, has lower emission per basic unit than that of C city. In case of D gun, in which the agricultural activities are active than the other two regions, the emission per basic unit is relatively much lower and in the aspect of the wastes characteristics, it is observed that its quality is lower, too. This is deemed to be caused by industrial structure, collection system, culture, lifestyle, etc. In the variation of wastes emission per basic unit by the time, no big difference was found among cities. After all, the variation of domestic 


\begin{tabular}{clccccc}
\hline \multicolumn{7}{c}{ MSW EMISSION IN C CITY, A CITY AND D GUN } \\
\hline \multirow{3}{*}{ C city } & \multicolumn{1}{c}{ TABLE-5 } \\
& Item & 2003 & 2004 & 2005 & 2006 & 2007 \\
& Population & 462.714 & 501.550 & 518.818 & 531.193 & 540.742 \\
& Emission (T/D) & 564 & 537 & 556 & 582 & 598 \\
& Unit emission (kg/day) & 1.219 & 1.071 & 1.072 & 1.096 & 1.106 \\
\hline \multirow{3}{*}{ A city } & Population & 193,122 & 200,938 & 208,448 & 217,112 & 227,815 \\
& Emission (T/D) & 194 & 207 & 216 & 229 & 223 \\
& Unit emission (kg/day) & 1.004 & 1.030 & 1.036 & 1.055 & 0.979 \\
\hline \multirow{3}{*}{ D gun } & Population & 118,701 & 117,409 & 122,016 & 127,167 & 138,796 \\
& Emission (T/D) & 77 & 77 & 85 & 76 & 78 \\
& Unit emission (kg/day) & 0.649 & 0.656 & 0.697 & 0.598 & 0.562 \\
\hline
\end{tabular}

wastes emission by region is deemed to have close relationship with the rate of population growth.

The future wastes emission calculated using mean wastes emission per basic unit by city is shown in Table-6. The wastes emission is increased as the population in all three regions are increased. Therefore, the much specific population growth rate and the wastes emission should be calculated by the years planned when planning the wastes treatment facilities and applied to basic design in order not to have problem in operation.

\begin{tabular}{cccccc}
\multicolumn{5}{c}{ TABLE-6 } \\
\multicolumn{5}{c}{$\begin{array}{c}\text { PREDICTION OF SOLID WASTE EMISSION } \\
\text { IN C CITY, A CITY AND D GUN }\end{array}$} \\
\hline Item & 2010 & 2015 & 2020 & 2025 \\
\hline C city & Emission (T/D) & 677 & 824 & 1,002 & 1,220 \\
A city & Emission (T/D) & 263 & 324 & 398 & 489 \\
D gun & Emission (T/D) & 99 & 121 & 147 & 179 \\
\hline
\end{tabular}

\section{Conclusion}

This study was conducted to seek effective wastes processing and management measures and to provide the basic data for establishment of the future wastes management plan by comparing and analyzing the physical and chemical characteristics of the domestic wastes in the small and medium sized cities of Chungcheongnam-do during winter season. The main results obtained in this study can be summarized as follows.

(1) The apparent densities of the three regions of C city, $A$ city and D gun were measured as $0.20,0.19,0.19$ ton $/ \mathrm{m}^{3}$ respectively and no distinctive regional characteristics were represented.

(2) In the physical composition, the foods contents were represented highest as 26.91 and $22.34 \%$ respectively and in case of D gun, the contents of the papers were represented high as $32.33 \%$ while the foods contents were represented relatively low as $15.37 \%$. Generally, it showed the trends that the contents of foods, papers and plastics were represented high.

(3) In the analysis results of three ingredients by region, the water contents were represented $34.69 \%$ in the C city while it represented 31.41 and $28.12 \%$ respectively in the A city and D gun and in the contents of combustibles, no big difference was represented by region as it was measures from 56.62 to $58.55 \%$.

(4) In the elementary analysis, the carbon content of the domestic wastes in D gun was $39.3 \%$, lower than A city of $57.16 \%$ and $\mathrm{C}$ city of $45.78 \%$ and the oxygen contents was $24.64 \%$, lower than A city and C city, which were measured from 29.36 to $30.03 \%$. The rests of elements of H, N, S, CI was measured by $6.13,1.13,0.05$ and $0.46 \%$ respectively in $\mathrm{C}$ city and in the A city, was measures by $6.85,0.76,0.05$ and $0.29 \%$ respectively, while in the D gun, they were represented as $5.67,0.06,0.22$ and $0.27 \%$ respectively and no significant difference was found.

(5) In the caloric value analysis results, which serves important factor in designing incinerator, the low caloric value of $\mathrm{C}$ city was represented highest as $2,634 \mathrm{kcal} / \mathrm{kg}$ while that of A city and D gun was analyzed as 2,231 kcal/kg and 2,188 $\mathrm{kcal} / \mathrm{kg}$, respectively.

(6) In the result of calculating wastes emission per basic unit by region, the $C$ city represented highest as $1.11 \mathrm{~kg} /$ day person, while A city represent as $1.02 \mathrm{~kg}$ /day person and the $\mathrm{D}$ gun represented as $0.63 \mathrm{~kg} /$ day person. The wastes emission per basic unit of the $\mathrm{C}$ city, which has highest population, was highest and $70 \%$ higher than the D gun, which represented the lowest calculation result.

\section{ACKNOWLEDGEMENTS}

This work was supported by the Human Resources Development of the Korea Insitute of Energy Technology Evaluation and Planning (KETEP) grant funded by the Korea Government Ministry of Knowledge Economy (No. 20114010203130).

\section{REFERENCES}

1. J.D. Chung, J.W. Kim and J.M. Han, J. Korea Soc. Waste Manage., 23, 344 (2006).

2. J.D. Chung and J.T. Kim, J. Korea Soc. Waste Manage., 21, 336 (2004).

3. Ministry of Environment: Wastes Process Test Methods (2007).

4. Ministry of Environment: National Wastes Generation and Processing Status (2008). 